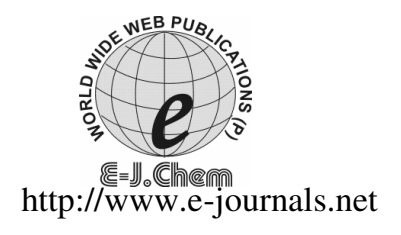

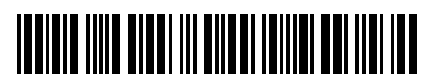

ISSN: 0973-4945; CODEN ECJHAO

E-Journal of Chemistry

2011, 8(3), 977-981

\title{
Experimental and Theoretical Evaluation of Ultrasonic Velocities of Binary Liquid Mixtures of Anisaldehyde and Toluene at Different Temperatures
}

\author{
CH.SRINIVASU, K.NARENDRA* and CH.KALPANA \\ Department of Physics, Andhra Loyola College \\ Vijayawada -8, Andhra Pradesh, India \\ *Department of Physics, V.R.Siddhartha Engg College \\ Vijayawada, Andhra Pradesh, India \\ kollanarendra@rediff.com
}

Received 31 August 2010; Accepted 8 November 2010

\begin{abstract}
Theoretical velocities of binary liquid mixtures of anisaldehyde with toluene at $303.15,308.15,313.15$ and $318.15 \mathrm{~K}$ have been evaluated by using theoretical models of liquid mixtures such as Nomoto, Van Dael-Vangeel, Schaff's collision factor theory and Junjie's relations. Density and ultrasonic velocity of these mixtures have also been measured as a function of concentration and temperature and the experimental values are compared with the theoretical values. A good agreement has been found between experimental and Nomoto's theoretical ultrasonic velocities. The results are explained in terms of intermolecular interactions occurring in these binary liquid mixtures.
\end{abstract}

Keywords: Theoretical ultrasonic velocities, Binary liquid mixtures, Anisaldehyde, Toluene

\section{Introduction}

Ultrasonic velocity in a medium is related to the binding forces between the molecules. Ultrasonic velocities in liquid mixtures consisting of polar and non-polar components are of considerable importance in understanding the intermolecular interaction between component molecules and find applications in several industrial and technological processes ${ }^{1-3}$. Several researchers ${ }^{4-7}$ carried out ultrasonic investigations on liquid mixtures and correlated the experimental results with the theoretical relations of Nomoto ${ }^{8}$, Van Dael and Vangeel ${ }^{9}$, Schaff' $s^{10}$ and Junjie's ${ }^{11}$ equations and interpreted the results in terms of molecular interactions. In the present paper ultrasonic velocity in binary mixtures from various theoretical models has been compared. The ultrasonic velocities of the chosen binary liquid mixture at $303.15,308.15,313.15$ and $318.15 \mathrm{~K}$ over the entire range of composition have been evaluated by using various theories and compared with the experimental values. 


\section{Experimental}

Anisaldehyde and toluene from Merk were purified using standard methods ${ }^{12,13}$. The purity of samples is checked by density and viscosity measurements. The densities of all the mixtures have been determined by using a single pan electrical balance for the determination of mass of a given volume of the liquid. The results of the densities are accurate to $\pm 0.5 \%$. Velocities were determined using single crystal ultrasonic pulse echo-interferometer (Model F-80X, Mittal enterprises, India) working at $2 \mathrm{MHz}$. The temperature of the solution was maintained constant by circulating water through jacket of double walled cell. All the measurements were made using constant temperature bath with in $\pm 0.01 \mathrm{~K}$.

\section{Results and Discussion}

Sound speeds were determined on the basis of different theories and relations as discussed earlier ${ }^{14}$ and the values are presented in Table 1 for the binary system at four different temperatures. The validity of different theoretical formulae is checked by percentage deviation at all the temperatures and is given in Table 2. The limitations and approximations incorporated in these theories are responsible for the deviations between theoretical and experimental values. When two liquids are mixed various types of forces are act due to interactions. Thus, the observed deviations between theoretical and experimental values of velocity shows that there is molecular interaction between the unlike molecules in the liquid mixture.

Table 1. Experimental and theoretical values of velocities in anisaldehyde + toluene system at different temperatures

\begin{tabular}{|c|c|c|c|c|c|}
\hline $\begin{array}{c}\text { Mole } \\
\text { fraction } \mathrm{x}\end{array}$ & $\begin{array}{l}\mathrm{U}_{\text {exp }} \\
\mathrm{ms}^{-1}\end{array}$ & $\begin{array}{c}\mathrm{U}_{\text {Nomoto }} \\
\mathrm{ms}^{-1}\end{array}$ & $\begin{array}{c}\mathrm{U}_{\text {idmix }} \\
\mathrm{ms}^{-1}\end{array}$ & $\begin{array}{l}\mathrm{U}_{\mathrm{CFT}} \\
\mathrm{ms}^{-1}\end{array}$ & $\begin{array}{c}\mathrm{U}_{\text {Junjiee }} \\
\mathrm{ms}^{-1}\end{array}$ \\
\hline \multicolumn{6}{|c|}{$303.15 \mathrm{~K}$} \\
\hline 0.0000 & 1694.33 & 1694.33 & 1694.33 & 1694.33 & 1597.29 \\
\hline 0.1151 & 1655.67 & 1651.60 & 1585.58 & 1645.84 & 1556.48 \\
\hline 0.2265 & 1644.67 & 1644.50 & 1505.92 & 1598.96 & 1521.63 \\
\hline 0.3342 & 1590.00 & 1544.89 & 1445.70 & 1553.62 & 1491.77 \\
\hline 0.4384 & 1530.66 & 1471.91 & 1399.26 & 1509.73 & 1466.15 \\
\hline 0.5394 & 1491.33 & 1506.33 & 1363.06 & 1467.24 & 1444.19 \\
\hline 0.6372 & 1444.33 & 1559.94 & 1334.71 & 1426.08 & 1425.41 \\
\hline 0.7321 & 1416.00 & 1364.97 & 1312.60 & 1386.17 & 1409.47 \\
\hline 0.8241 & 1368.00 & 1369.86 & 1295.56 & 1347.48 & 1396.05 \\
\hline 0.9133 & 1329.00 & 1451.77 & 1282.74 & 1309.94 & 1384.92 \\
\hline 1.0000 & 1273.50 & 1273.50 & 1273.50 & 1273.50 & 1375.89 \\
\hline \multicolumn{6}{|c|}{$308.15 \mathrm{~K}$} \\
\hline 0.0000 & 1670.67 & 1670.67 & 1670.67 & 1670.67 & 1584.59 \\
\hline 0.1151 & 1640.67 & 1671.77 & 1565.89 & 1623.75 & 1545.80 \\
\hline 0.2265 & 1583.33 & 1653.92 & 1488.86 & 1578.40 & 1512.41 \\
\hline 0.3342 & 1530.00 & 1564.56 & 1430.50 & 1534.53 & 1483.60 \\
\hline 0.4384 & 1482.00 & 1492.17 & 1385.45 & 1492.07 & 1458.71 \\
\hline 0.5394 & 1434.00 & 1518.25 & 1350.29 & 1450.96 & 1437.22 \\
\hline 0.6372 & 1419.00 & 1573.56 & 1322.76 & 1411.13 & 1418.72 \\
\hline 0.7321 & 1386.67 & 1374.18 & 1301.30 & 1372.52 & 1402.88 \\
\hline 0.8241 & 1325.00 & 1368.77 & 1284.78 & 1335.08 & 1389.44 \\
\hline 0.9133 & 1304.00 & 1443.02 & 1272.39 & 1298.76 & 1378.17 \\
\hline 1.0000 & 1263.50 & 1263.50 & 1263.50 & 1263.50 & 1368.91 \\
\hline
\end{tabular}




\begin{tabular}{cccccc}
\hline $\begin{array}{c}\text { Mole } \\
\text { fraction x }\end{array}$ & $\begin{array}{c}\mathrm{U}_{\text {exp }} \\
\mathrm{ms}^{-1}\end{array}$ & \begin{tabular}{c}
$\mathrm{U}_{\text {Nomoto }} \mathrm{ms}^{-1}$ \\
\multicolumn{5}{c}{$313.15 \mathrm{~K}$}
\end{tabular} & $\begin{array}{c}\mathrm{U}_{\text {idmix }} \\
\mathrm{ms}^{-1}\end{array}$ & $\begin{array}{c}\mathrm{U}_{\mathrm{CFT}} \\
\mathrm{ms}^{-1}\end{array}$ & $\begin{array}{c}\mathrm{U}_{\text {Junjiee }} \\
\mathrm{ms}^{-1}\end{array}$ \\
\hline 0.0000 & 1631.33 & 1631.33 & 1631.33 & 1631.33 & 1548.74 \\
0.1151 & 1626.67 & 1644.64 & 1535.06 & 1587.80 & 1514.50 \\
0.2265 & 1569.67 & 1616.87 & 1463.69 & 1545.71 & 1484.94 \\
0.3342 & 1494.99 & 1530.52 & 1409.31 & 1505.00 & 1459.40 \\
0.4384 & 1416.00 & 1454.92 & 1367.19 & 1465.61 & 1437.33 \\
0.5394 & 1404.00 & 1500.91 & 1334.26 & 1427.46 & 1418.31 \\
0.6372 & 1398.00 & 1547.17 & 1308.49 & 1390.50 & 1401.98 \\
0.7321 & 1353.00 & 1360.59 & 1288.43 & 1354.67 & 1388.07 \\
0.8241 & 1315.00 & 1366.63 & 1273.05 & 1319.93 & 1376.36 \\
0.9133 & 1293.00 & 1419.43 & 1261.60 & 1286.22 & 1366.66 \\
1.0000 & 1253.50 & 1253.50 & 1253.50 & 1253.50 & 1358.81 \\
& & $318.15 \mathrm{~K}$ & & \\
0.0000 & 1557.00 & 1557.00 & 1557.00 & 1557.00 & 1481.38 \\
0.1151 & 1544.33 & 1575.39 & 1476.74 & 1520.08 & 1456.19 \\
0.2265 & 1499.00 & 1556.48 & 1416.24 & 1484.38 & 1434.30 \\
0.3342 & 1442.00 & 1472.97 & 1369.66 & 1449.85 & 1415.33 \\
0.4384 & 1386.00 & 1422.08 & 1333.35 & 1416.43 & 1398.93 \\
0.5394 & 1366.67 & 1458.69 & 1304.90 & 1384.07 & 1384.85 \\
0.6372 & 1332.67 & 1522.06 & 1282.66 & 1352.72 & 1372.88 \\
0.7321 & 1317.99 & 1333.97 & 1265.46 & 1322.33 & 1362.82 \\
0.8241 & 1300.00 & 1331.58 & 1252.43 & 1292.85 & 1354.53 \\
0.9133 & 1269.00 & 1367.98 & 1242.94 & 1264.26 & 1347.90 \\
1.0000 & 1236.50 & 1236.50 & 1236.50 & 1236.50 & 1342.83 \\
\hline
\end{tabular}

It is observed from Table 2 that there is a good agreement between experimental and theoretical values calculated by Nomoto's relation followed by CFT while higher deviations are observed in Junjie's relation and Van Dael ideal mixing relation.

Table 2. Percentage deviation between experimental and theoretical values of velocities in anisaldehyde + toluene system at different temperatures

\begin{tabular}{ccccc}
\hline Mole fraction $x$ & $\begin{array}{c}\% \mathrm{U}_{\text {Nomoto }} \\
\mathrm{ms}^{-1}\end{array}$ & $\begin{array}{c}\% \mathrm{U}_{\text {imx }} \\
\mathrm{ms}^{-1}\end{array}$ & $\begin{array}{c}\% \mathrm{U}_{\text {CFT }} \\
\mathrm{ms}^{-1}\end{array}$ & $\begin{array}{c}\% \mathrm{U}_{\text {Junjiee }} \\
\mathrm{ms}^{-1}\end{array}$ \\
\hline & & $303.15 \mathrm{~K}$ & & \\
0.0000 & 0.0000 & 0.0000 & 0.0000 & 5.7275 \\
0.1151 & 0.2456 & 4.2331 & -0.5937 & 5.9909 \\
0.2265 & 0.0102 & 8.4366 & -2.7793 & 7.4810 \\
0.3342 & 2.8372 & 9.0758 & -2.2881 & 6.1779 \\
0.4384 & 3.8385 & 8.5843 & -1.3674 & 4.2145 \\
0.5394 & -1.0058 & 8.6012 & -1.6153 & 3.1613 \\
0.6372 & -8.0046 & 7.5894 & -1.2636 & 1.3097 \\
0.7321 & 3.6041 & 7.3020 & -2.1066 & 0.4613 \\
\hline
\end{tabular}




\begin{tabular}{|c|c|c|c|c|}
\hline Mole fraction $\mathrm{x}$ & $\begin{array}{c}\% \mathrm{U}_{\text {Nomoto }} \\
\mathrm{ms}^{-1}\end{array}$ & $\begin{array}{c}\% \mathrm{U}_{\mathrm{imx}} \\
\mathrm{ms}^{-1}\end{array}$ & $\begin{array}{c}\% \mathrm{U}_{\mathrm{CFT}} \\
\mathrm{ms}^{-1}\end{array}$ & $\begin{array}{c}\% \mathrm{U}_{\text {Junjiee }} \\
\mathrm{ms}^{-1}\end{array}$ \\
\hline 0.8241 & -0.1361 & 5.2953 & -1.5000 & -2.0506 \\
\hline 0.9133 & -9.2377 & 3.4810 & -1.4342 & -4.2080 \\
\hline 1.0000 & 0.0000 & 0.0000 & 0.0000 & -8.0402 \\
\hline \multicolumn{5}{|c|}{$308.15 \mathrm{~K}$} \\
\hline 0.0000 & 0.0000 & 0.0000 & 0.0000 & 5.1525 \\
\hline 0.1151 & -1.8958 & 4.5582 & -1.0313 & 5.7826 \\
\hline 0.2265 & -4.4583 & 5.9667 & -0.3114 & 4.4789 \\
\hline 0.3342 & -2.2591 & 6.5031 & 0.2961 & 3.0325 \\
\hline 0.4384 & -0.6862 & 6.5151 & 0.6795 & 1.5717 \\
\hline 0.5394 & -5.8750 & 5.8376 & 1.1827 & -0.2243 \\
\hline 0.6372 & -10.8919 & 6.7819 & -0.5546 & 0.0199 \\
\hline 0.7321 & 0.9008 & 6.1562 & -1.0204 & -1.1690 \\
\hline 0.8241 & -3.3033 & 3.0351 & 0.7608 & -4.8633 \\
\hline 0.9133 & -10.6612 & 2.4243 & -0.4018 & -5.6882 \\
\hline 1.0000 & 0.0000 & 0.0000 & 0.0000 & -8.3427 \\
\hline \multicolumn{5}{|c|}{$313.15 \mathrm{~K}$} \\
\hline 0.0000 & 0.0000 & 0.0000 & 0.0000 & 5.0628 \\
\hline 0.1151 & -1.1050 & 5.6316 & -2.3895 & 6.8959 \\
\hline 0.2265 & -3.0068 & 6.7520 & -1.5264 & 5.3976 \\
\hline 0.3342 & -2.3764 & 5.7310 & 0.6696 & 2.3804 \\
\hline 0.4384 & -2.7485 & 3.4472 & 3.5035 & -1.5066 \\
\hline 0.5394 & -6.9021 & 4.9669 & 1.6709 & -1.0190 \\
\hline 0.6372 & -10.6702 & 6.4028 & -0.5365 & -0.2846 \\
\hline 0.7321 & -0.5609 & 4.7726 & 0.1234 & -2.5922 \\
\hline 0.8241 & -3.9259 & 3.1902 & 0.3749 & -4.6662 \\
\hline 0.9133 & -9.7784 & 2.4287 & -0.5244 & -5.6966 \\
\hline 1.0000 & 0.0000 & 0.0000 & 0.0000 & -8.4015 \\
\hline \multicolumn{5}{|c|}{$318.15 \mathrm{~K}$} \\
\hline 0.0000 & 0.0000 & 0.0000 & 0.0000 & 4.8568 \\
\hline 0.1151 & -2.0111 & 4.3765 & -1.5703 & 5.7076 \\
\hline 0.2265 & -3.8348 & 5.5211 & -0.9753 & 4.3160 \\
\hline 0.3342 & -2.1478 & 5.0169 & 0.5444 & 1.8497 \\
\hline 0.4384 & -2.6035 & 3.7990 & 2.1955 & -0.9331 \\
\hline 0.5394 & -6.7330 & 4.5197 & 1.2732 & -1.3306 \\
\hline 0.6372 & -14.2115 & 3.7523 & 1.5045 & -3.0169 \\
\hline 0.7321 & -1.2124 & 3.9855 & 0.3293 & -3.4012 \\
\hline 0.8241 & -2.4295 & 3.6590 & -0.5500 & -4.1949 \\
\hline 0.9133 & -7.7997 & 2.0536 & -0.3735 & -6.2179 \\
\hline 1.0000 & 0.0000 & 0.0000 & 0.0000 & -8.5995 \\
\hline
\end{tabular}




\section{Conclusion}

Out of four theories and relations, the Nomoto's relation provides the best results. The Success of Nomoto's relation in predicting the experimental ultrasonic velocities for liquid mixtures has been supported by others ${ }^{15,16}$.

\section{References}

1. Valen A, Lopez M C, Urieta J S, Royo F M and Lafuente C, J Mol Liq., 2002, 95, 157-165.

2. Pal A and Kumar H, J Chem Thermodyn, 2004, 36, 173.

3. Rao T S, Veeraiah N and Rambabu C, Indian J Pure Appl Phys., 2002, 40, 850.

4. Suresh reddy K V N, Srinivasa Rao P and Krishnaiah A, Phys and Chem of Liq., 2004, 42(6), 561-588.

5. Rama Rao G V, Sandhya Sri P B, Viswanatha Sarma A and Rambabu C, Indian J Pure Appl Phys., 2007, 45(2), 135-142.

6. $\quad$ Sundharam N and Palaniappan L, Indian J Phys., 2005, 79, 1173-1178.

7. Ali A Abida, Nain A K and Hyder S, Indian J Pure Appl Ultrason, 2001, 23, 73.

8. Nomoto O, J Phys Soc Japan, 1949, 4, 280 and 1958, 13, 1528.

9. Van Dael W and Vangeel E, Proc Int Conf on Calorimetry and Thermodynamics, Warasa 1955, 555.

10. Schaffs W, Acustica, 1975, 33, 272.

11. Junjie Z, J China Univ Sci Techn, 1984, 14, 298.

12. Vogel A I, Textbook of Organic Chemistry, $5^{\text {th }}$ Ed., John Wiley: New York, 1989.

13. Riddick J A, Bunger W B and Sakano T K, Organic Solvents, $4^{\text {th }}$ Ed., John Willey and Sons, New York, 1986.

14. Rita Mehra and Avneesh K. Gaur, J Chem Eng Data, 2008, 53, 863.

15. Kaulgud M V, Acustica, 1960, 10, 316.

16. Meyyappan M and Sethupathy, Indian J Pure Appl Ultrason., 2003, 25, 1. 


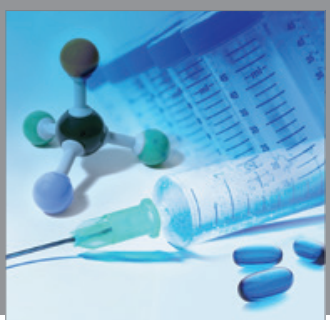

International Journal of

Medicinal Chemistry

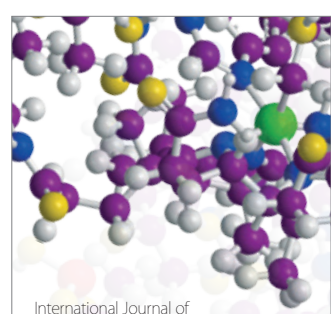

Carbohydrate Chemistry

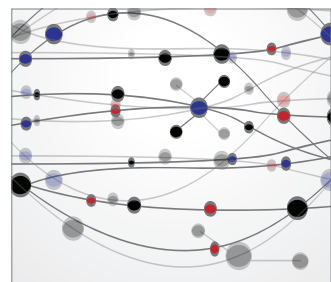

The Scientific World Journal
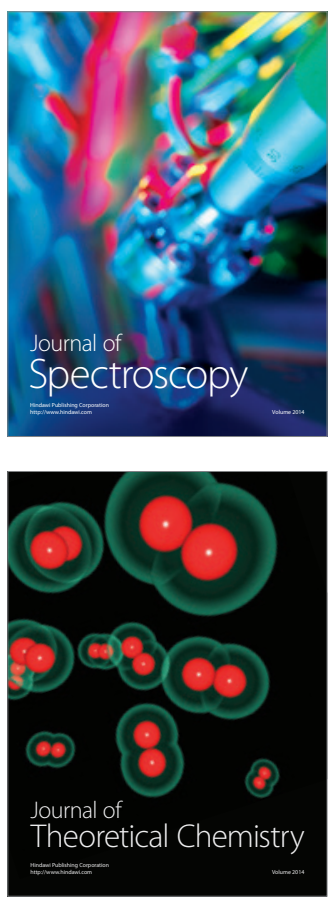
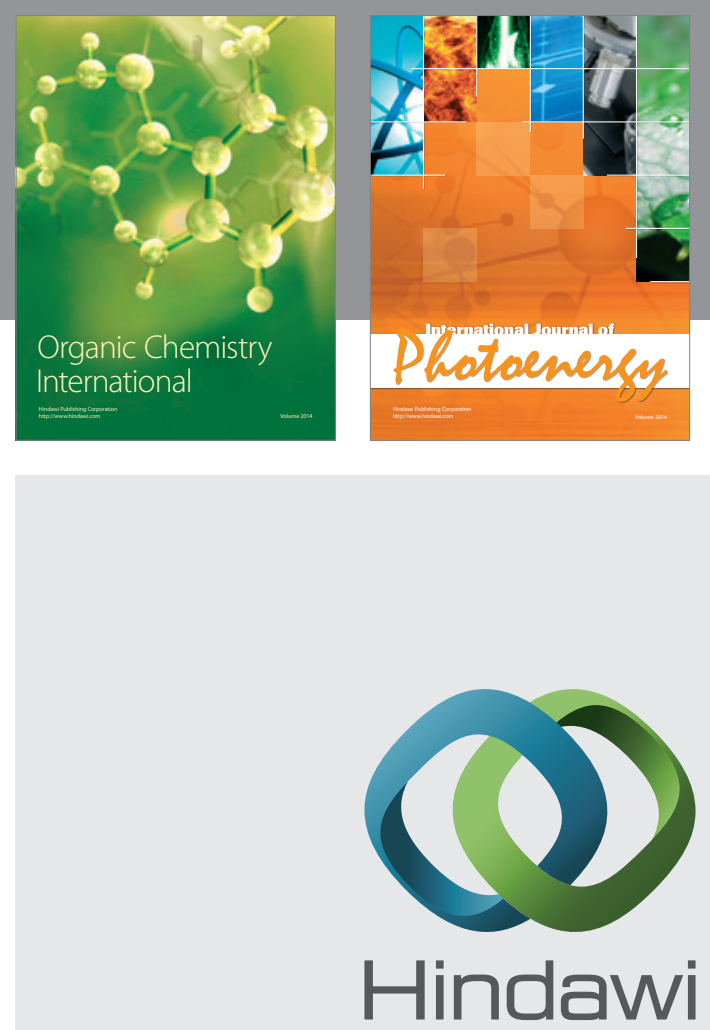

Submit your manuscripts at

http://www.hindawi.com
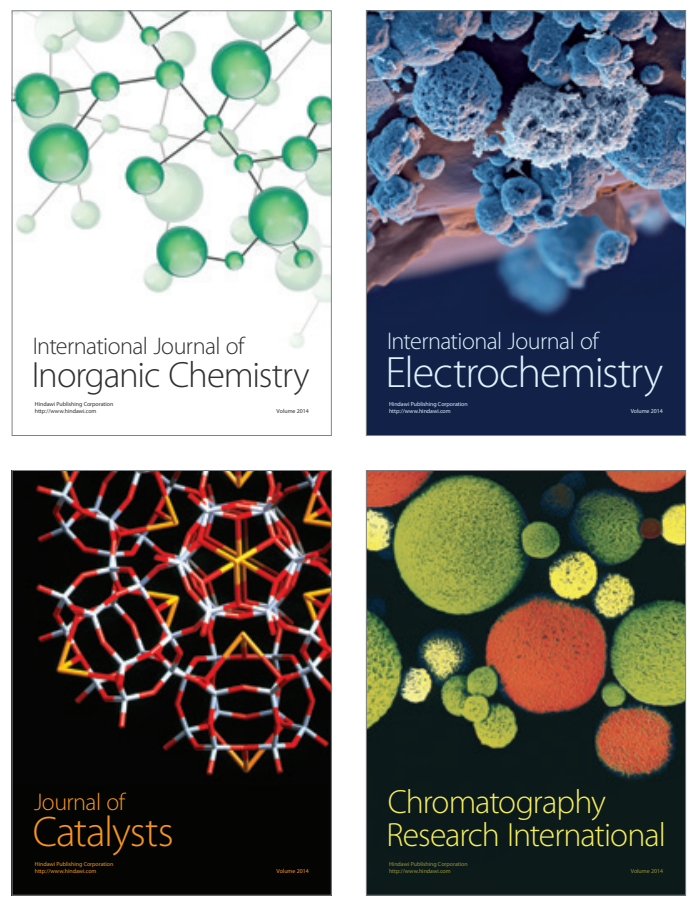
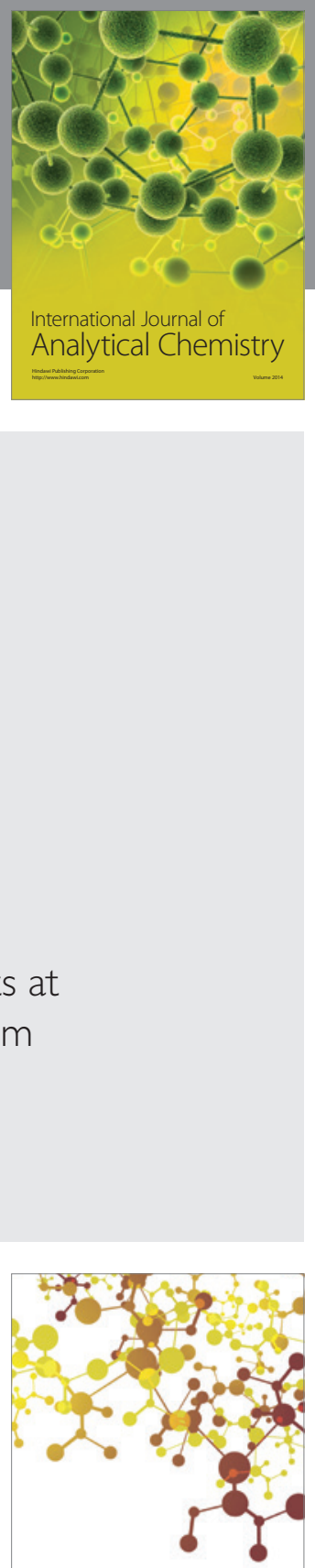

Journal of

Applied Chemistry
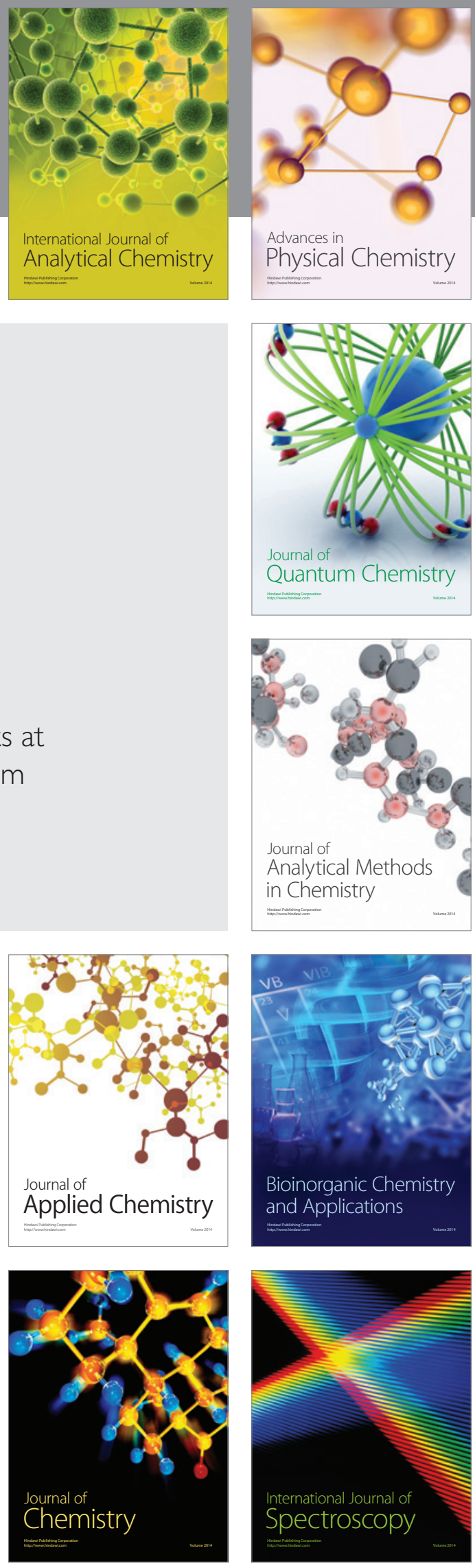